\title{
NONEXISTENCE OF POSITIVELY EXPANSIVE MAPS ON COMPACT CONNECTED MANIFOLDS WITH BOUNDARY
}

\author{
KOICHI HIRAIDE \\ (Communicated by Dennis Burke) \\ Dedicated to Professor Ryosuke Nakagawa on his sixtieth birthday
}

\begin{abstract}
In this note we prove that no compact connected manifold with boundary admits a positively expansive map.
\end{abstract}

Let $(X, d)$ be a compact metric space and $f: X \rightarrow X$ be a continuous map (not always surjective). We say that $f$ is positively expansive if there is a constant $c>0$ such that if $x, y \in X$ and $x \neq y$ then $d\left(f^{i}(x), f^{i}(y)\right)>c$ for some $i \geq 0$ ( $c$ is called an expansive constant for $f$ ). The notion of positive expansiveness is independent of the metrics compatible with original topology, and preserved under topological conjugacy. In D. W. Curtis and S. Miklos [3] they proved that no positively expansive map of $X$ onto $X$ can be a homeomorphism if $X$ is nontrivial and connected. In fact, whenever $X$ admits a positively expansive homeomorphism $f, X$ is finite (cf. [1,5]). This is easily checked as follows. By a result of W. L. Reddy [10], $f$ is expanding with respect to some metric $\rho$ for $X$, i.e. there exist constants $\delta>0$ and $0<\lambda<1$ such that $\rho(x, y)<\delta$ implies $\rho\left(f^{-1}(x), f^{-1}(y)\right) \leq \lambda \rho(x, y)$. Thus $\Phi^{-}=\left\{f^{-i}: i \geq 0\right\}$ is uniformly equicontinuous. By a metric $D$ defined by $D(f, g)=\max \{\rho(f(x), g(x)): x \in X\}, \Phi^{-}$is totally bounded. Let $\Phi^{+}=$ $\left\{f^{i}: i \geq 0\right\}$ and define a map $G: \Phi^{-} \rightarrow \Phi^{+}$by $G\left(f^{-i}\right)=f^{i}$ for $i \geq 0$. Then $G$ is $D$-isometric. Therefore $\Phi^{+}$is totally bounded. Since $X$ is compact, $\Phi^{+}$is uniformly equicontinuous and so there is $\varepsilon>0$ such that $\rho(x, y)<\varepsilon$ implies $\rho\left(f^{i}(x), f^{i}(y)\right)<c$ for all $i \geq 0$ ( $c$ is an expansive constant for $f$ ). This shows $x=y$ and therefore $x$ is an isolated point.

The study of positively expansive maps is an interesting subject in topological dynamics. In [3] the following is posed: "Characterize all compact connected manifolds which admit positively expansive maps." Our aim is to give an answer for this problem.

Received by the editors May 22, 1989 and, in revised form, October 6, 1989.

1980 Mathematics Subject Classification (1985 Revision). Primary 54E40, 54H20, 58F15; Secondary $34 \mathrm{C} 35$.

Key words and phrases. Positively expansive map, manifold with boundary, infra-nil-manifold. 
Theorem 1. No compact connected manifold with boundary admits a positively expansive map.

M. Shub [11, 12], J. Franks [4], M. W. Hirsch [9], and M. Gromov [6] studied the problem of characterization of expanding differentiable maps on closed smooth manifolds. In [6] M. Gromov proved finally that an expanding differentiable map of an arbitrary closed smooth manifold is topologically conjugate to an expanding infra-nil-endomorphism. On the other hand, E. M. Coven and W. L. Reddy [2] studied positively expansive maps of closed topological manifolds and showed that such maps are expanding with respect to certain metrics. Recently the author [8] generalized a result of M. Gromov [6] as follows: a positively expansive map of an arbitrary closed topological manifold is topologically conjugate to an expanding infra-nil-endomorphism. Combining this and Theorem 1, we can conclude the following

Theorem 2. Every compact connected manifold which admits a positively expansive map is homeomorphic to an infra-nil-manifold, and such a map of an infranil-manifold is topologically conjugate to an expanding infra-nil-endomorphism.

Theorem 1 will be obtained by using the following.

Lemma. Let $X$ be a compact connected locally connected metric space and $f: X \rightarrow X$ be a positively expansive map. If a closed proper subset $K$ of $X$ satisfies the following conditions:

(1) $f(X \backslash K) \subset X \backslash K$,

(2) $f_{\mid X \backslash K}: X \backslash K \rightarrow X \backslash K$ is an open map,

then $K=\phi$ (compare with Theorem 3 of [3]).

To use the lemma for the proof of Theorem 1, let $M$ be a compact connected manifold and $\partial M$ denote the boundary of $M$. Suppose that $M$ admits a positively expansive map $f$. From the definition it follows that $f$ is locally injective. Combining this fact and Brouwer's theorem on invariance of domain, we have that $f(M \backslash \partial M) \subset M \backslash \partial M$ and $f_{\mid M \backslash \partial M}: M \backslash \partial M \rightarrow M \backslash \partial M$ is an open map. Use the lemma here, then we have $\partial M=\phi$.

We must prove the lemma to obtain the conclusion. Let $f: X \rightarrow X$ be as in the lemma. Then there exist a metric $\rho$ for $X$ and constants $\delta>0$ and $\lambda>1$ such that if $\rho(x, y)<\delta$ then $\rho(f(x), f(y)) \geq \lambda \rho(x, y)$. This is proved in the same way as in [10] (notice that $f$ is not always surjective). For $\epsilon>0$ and $x \in X$, let $U_{\epsilon}(x)=\{y \in X: \rho(x, y)<\epsilon\}$ and denote by $C_{\epsilon}(x)$ the connected component of $x$ in $U_{\epsilon}(x)$. Obviously $C_{\epsilon}(x)$ is open in $X$. Since $X$ is locally arcwise connected (Theorem 6.29 of [7]), we have that $C_{\epsilon}(x)$ is arcwise connected.

We first check the following claim: Let $0<\epsilon<\delta / 2$ and $x \in X \backslash K$. If $C_{\epsilon}(x) \subset X \backslash K$, then $f\left(C_{\epsilon}(x)\right) \supset C_{\lambda \epsilon}(f(x))$.

Assume $y \in C_{\lambda \epsilon}(f(x)) \backslash f\left(C_{\epsilon}(x)\right) \neq \phi$. Since $C_{\lambda \epsilon}(f(x))$ is arcwise connected, there exists an arc $\omega:[0,1] \rightarrow C_{\lambda \epsilon}(f(x))$ such that $\omega(0)=f(x)$ and $\omega(1)=y$. 
Since $C_{\epsilon}(x) \subset X \backslash K, f\left(C_{\epsilon}(x)\right)$ is open in $X$ by (2). Obviously $\omega(0)=f(x) \epsilon$ $f\left(C_{\epsilon}(x)\right)$. Hence we can take $0<t_{0}<1$ such that $\omega\left(\left[0, t_{0}\right)\right) \subset f\left(C_{\epsilon}(x)\right)$ and $\omega\left(t_{0}\right) \notin f\left(C_{\epsilon}(x)\right)$. Then $\omega\left(\left[0, t_{0}\right]\right) \subset \overline{f\left(C_{\epsilon}(x)\right)}$ where $\overline{f\left(C_{\epsilon}(x)\right)}$ denotes the closure of $f\left(C_{\epsilon}(x)\right)$ in $X$.

Obviously $f\left(\overline{C_{\epsilon}(x)}\right)=\overline{f\left(C_{\epsilon}(x)\right)}$. Since $\overline{C_{\epsilon}(x)} \subset U_{\delta / 2}(x)$, by the choice of $\delta$ we have that $\left.f\right|_{\overline{C_{\epsilon}(x)}}: \overline{C_{\epsilon}(x)} \rightarrow \overline{f\left(C_{\epsilon}(x)\right)}$ is injective, and so $\left.f\right|_{\overline{C_{\epsilon}(x)}}: \overline{C_{\epsilon}(x)} \rightarrow$ $\overline{f\left(C_{\epsilon}(x)\right)}$ is a homeomorphism. Since $\omega\left(\left[0, t_{0}\right]\right) \subset \overline{f\left(C_{\epsilon}(x)\right)}$, we can find an $\operatorname{arc} \bar{\omega}:\left[0, t_{0}\right] \rightarrow \overline{C_{\epsilon}(x)}$ such that $f \circ \bar{\omega}=\omega$. Note that $\omega\left(\left[0, t_{0}\right)\right) \subset f\left(C_{\epsilon}(x)\right)$. Then we have that $\bar{\omega}\left(\left[0, t_{0}\right)\right) \subset C_{\epsilon}(x)$. Since $\omega\left(t_{0}\right) \notin f\left(C_{\epsilon}(x)\right)$, obviously $\omega\left(t_{0}\right) \notin C_{\epsilon}(x)$.

On the other hand, since $\omega\left(t_{0}\right) \in C_{\lambda \epsilon}(f(x))$, we have

$$
\lambda \rho\left(\bar{\omega}\left(t_{0}\right), x\right) \leq \rho\left(\omega\left(t_{0}\right), f(x)\right)<\lambda \epsilon,
$$

and so $\bar{\omega}\left(t_{0}\right) \in U_{\epsilon}(x)$. Combining this and the fact that $x \in \bar{\omega}\left(\left[0, t_{0}\right)\right) \subset$ $C_{\epsilon}(x) \subset U_{\epsilon}(x)$, we have that $\bar{\omega}\left(t_{0}\right) \in C_{\epsilon}(x)$, thus a contradiction.

We proceed to the proof of the lemma. For $\epsilon>0$ let

$$
X(\epsilon)=\left\{x \in X \backslash K: C_{\epsilon}(x) \subset X \backslash K\right\} .
$$

Since $K$ is a closed proper subset of $X$, there exist $0<\epsilon_{0}<\delta / 2$ such that $X\left(\epsilon_{0}\right) \neq \phi$. Assume that $K \neq \phi$. Obviously $X\left(\epsilon_{0}\right) \varsubsetneqq X$. Let $x \in X\left(\epsilon_{0}\right)$. Then $C_{\epsilon_{0}}(x) \subset X \backslash K$. From the above claim $f\left(C_{\epsilon_{0}}(x)\right) \supset C_{\lambda \epsilon_{0}}(f(x))$. Hence $C_{\lambda \epsilon_{0}}(f(x)) \subset X \backslash K$ by (1) and so $f(x) \in X\left(\lambda \epsilon_{0}\right)$. Therefore $f\left(X\left(\epsilon_{0}\right)\right) \subset$ $X\left(\lambda \epsilon_{0}\right)$.

It is easily checked that $X\left(\lambda \epsilon_{0}\right) \subset X\left(\mu \epsilon_{0}\right) \subset X\left(\epsilon_{0}\right)$ for $1<\mu<\lambda$. We show that $\overline{X\left(\lambda \epsilon_{0}\right)} \subset X\left(\mu \epsilon_{0}\right)$. To do this, let $\left\{x_{i}\right\}_{i \geq 0}$ be a sequence of $X\left(\lambda \epsilon_{0}\right)$ and let $x_{i} \rightarrow x \in X$ as $i \rightarrow \infty$. Obviously $U_{\mu \epsilon_{0}}(x) \subset U_{\lambda \epsilon_{0}}\left(x_{i}\right)$ for sufficiently large $i$. Since $C_{\mu \epsilon_{0}}(x)$ is oepn in $X$, we may assume that $x_{i} \in C_{\mu \epsilon_{0}}(x)$. Then $C_{\mu \epsilon_{0}}(x) \subset C_{\lambda \epsilon_{0}}\left(x_{i}\right)$, which implies $x \in X\left(\mu \epsilon_{0}\right)$. Therefore $\overline{X\left(\lambda \epsilon_{0}\right)} \subset X\left(\mu \epsilon_{0}\right)$.

Consequently we have

$$
f\left(\overline{X\left(\epsilon_{0}\right)}\right)=\overline{f\left(X\left(\epsilon_{0}\right)\right)} \subset \overline{X\left(\lambda \epsilon_{0}\right)} \subset X\left(\mu \epsilon_{0}\right) \subset X\left(\epsilon_{0}\right) .
$$

Hence $Y=\bigcap_{i \geq 0} f^{i}\left(\overline{X\left(\epsilon_{0}\right)}\right)$ is a nonempty closed set and $f(Y)=Y$. Since $Y \subset X\left(\epsilon_{0}\right) \varsubsetneqq X$ and $X$ is connected, obviously $Y$ is not open in $X$.

For $A \subset X$ and $\alpha>0$ let $N_{\alpha}(A)=\bigcup_{a \in A} C_{\alpha}(a)$. Then we have $N_{(\mu-1) \epsilon_{0}}(Y)$ $\subset X\left(\epsilon_{0}\right)$. This is checked as follows. Let $z \in Y$ and $x \in C_{(\mu-1) \epsilon_{0}}(z)$. Then $C_{\epsilon_{0}}(x) \subset C_{\mu \epsilon_{0}}(z)$. Since $z \in Y \subset X\left(\mu \epsilon_{0}\right), C_{\epsilon_{0}}(x) \subset C_{\mu \epsilon_{0}}(z) \subset X \backslash K$ and so $x \in X\left(\epsilon_{0}\right)$.

By the above result $Y \subset N_{(\mu-1) \epsilon_{0}}(Y) \subset \overline{X\left(\epsilon_{0}\right)}$. Since $f(Y)=Y$, we have

$$
Y \subset \bigcap_{i \geq 0} f^{i}\left(N_{(\mu-1) \epsilon_{0}}(Y)\right) \subset \bigcap_{i \geq 0} f^{i}\left(\overline{X\left(\epsilon_{0}\right)}\right)=Y,
$$

and hence $Y=\bigcap_{i \geq 0} f^{i}\left(N_{(\mu-1) \epsilon_{0}}(Y)\right)$. 
On the other hand, let $z \in Y$. Then $C_{(\mu-1) \epsilon_{0}}(z) \subset C_{\mu \epsilon_{0}}(z) \subset X \backslash K$. From the claim we have $f\left(C_{(\mu-1) \epsilon_{0}}(z)\right) \supset C_{\lambda(\mu-1) \epsilon_{0}}(f(z))$. Hence $f\left(N_{(\mu-1) \epsilon_{0}}(Y)\right) \supset$ $N_{\lambda(\mu-1) \epsilon_{0}}(Y) \supset N_{(\mu-1) \epsilon_{0}}(Y)$ (since $f(Y)=Y$.) Therefore $Y=N_{(\mu-1) \epsilon_{0}}(Y)$. This implies that $Y$ is open in $X$. We arrived at a contradiction.

\section{REFERENCES}

1. N. Aoki and K. Hiraide, Dynamical systems of self-covering maps of the tori, preprint.

2. E. M. Coven and W. L. Reddy, Positively expansive maps of compact manifolds, Lecture Notes in Math., vol. 819, Springer-Verlag, Berlin and New York, 1980, pp. 96-110.

3 D. W. Curtis and S. Miklos, Nonexistence of local expansions on certain continua, Fund. Math. 129 (1988), 207-210.

4. J. Franks, Anosov diffeomorphisms, Global Anal., Proc. Sympos. Pure Math., vol. 14, Amer. Math. Soc., Providence, RI, 1970, pp. 61-93.

5. W. H. Gottschalk and G. A. Hedlund, Topological dynamics, Amer. Math. Soc. Colloq, Publ., vol. 36, Amer. Math. Soc., Providence, RI, 1955.

6. M. Gromov, Groups of polynomial growth and expanding maps, Inst. Hautes Études Sci. Publ. Math. 53 (1981), 53-78.

7. D. W. Hall and G. L. Spencer II, Elementary topology, Wiley, New York, 1955.

8. K. Hiraide, Positively expansive maps and growth of fundamental groups, Proc. Amer. Math. Soc. 104 (1988), 934-941.

9. M. W. Hirsch, Expanding maps and transformation groups, Global Anal., Proc. Sympos. Pure Math., vol. 14, Amer. Math. Soc., Providence, RI, 1970, pp. 125-131.

10. W. L. Reddy, Expanding maps on compact metric spaces, Topology Appl. 13 (1982), 327334.

11. M. Shub, Endomorphisms of compact differentiable manifolds, Amer. J. Math. 91 (1969), 175-199.

12. __ Expanding maps, Global Anal., Proc. Sympos. Pure Math., vol. 14, Amer. Math. Soc., Providence, RI, 1970, pp. 273-276.

Institute of Mathematics, University of Tsukuba, Ibaraki 305, Japan 